\title{
CÓLICO NEFRÍTICO EN EL SERVICIO DE URGENCIAS. ESTUDIO EPIDEMIOLÓGICO, DIAGNÓSTICO Y ETIOPATOGÉNICO
}

\author{
José A. Hermida Pérezl, Mํa de La Paz Pérez Palmes², Juan Francisco Loro Ferrer³, Otto Ochoa \\ Urdangarain 4 y Abdel Buduen Nuñez.
}

\begin{abstract}
'Servicio de Urgencias de Los Llanos de Aridane. Sta Cruz de Tenerife. Canarias. España.
${ }^{2}$ Atención Primaria. Centro de Salud de lcod de los Vinos. Sta Cruz de Tenerife. Canarias. España.

${ }^{3}$ Departamento Ciencias Clínicas. Facultad de Medicina. ULPGC. Universidad de Las Palmas de Gran Canaria.

4Hospital Provincial Docente "Manuel Ascunce Domenech". Camagüey. Cuba.

5 Técnico en Radiodiagnóstico.
\end{abstract}

Resumen.- OBJETIVO: Estudiar los factores epidemiológicos, etiopatogénicos y clínicos del cólico nefrítico (CN).

MÉTODO/RESULTADOS: Se realizó un estudio prospectivo, multicéntrico, transversal con 146 pacientes $(n=146)$ tratados en el Servicio de Urgencias por CN y 146 casos control sin CN (n=146). Se valoraron: edad, sexo, antecedentes patológicos, factores etiopatogénicos de la litiasis renal, cuadro clínico, urianálisis, composición química de la litiasis y radiología. Se hizo un análisis comparativo estadístico (hallazgo p) entre ambos grupos de pacientes. El CN fue más frecuente en varones, el pico máximo de incidencia fue de $31-50$ años, con $36,30 \%$ en hombres y $21,23 \%$ de

\section{CORRESPONDENCIA}

José A. Hermida Pérez

Avda.Carlos Francisco Lorenzo Navarro, 56 3ํ A Los llanos de Aridane

Sta Cruz de Tenerife, 38760 Canarias. (España).

hermidana@yahoo.es

Aceptado para publicar: 21 de mayo 2009 mujeres, el 60,27\% catalogaron el dolor como intensidad severa, 140 pacientes con CN $195,89 \%)$ presentaron antecedentes urológicos vs. $15(10,27 \%)$ en los $\sin C N(p=0,001)$, fue más frecuente la presentación con dolor intenso, brusco, lumboabdominal o lumbar $(93,83 \%)$, tenían historia familiar de enfermedad litiásica 23,28\% de pacientes con CN vs. 6, 16\% en los $\sin C N(p<0,001)$, el mayor número de $C N$ se vio en los meses de verano (36,58\%), 80,82\% de pacientes con CN ingerían < de $2 \mathrm{~L}$ agua/día vs. 18,49\% en los $\sin C N(p<0,001)$. Hubo hematuria en un $90,41 \%$ de CN vs. $11,64 \%$ de los $\sin C N(p<0,001)$. Se visualizó litiasis $(R x)$ en un $42,10 \%$ vs. $57,89 \%$ donde no se visualizó. La litiasis más frecuente fue la de oxalato cálcico mono o dihidratado $(61,2 \%)$.

CONCLUSIONES: La incidencia de CN en nuestra área de salud es mayor en el sexo masculino. El dolor característico del CN es agudo, severo, de aparición súbita, localizado en la región lumbar, por debajo de las costillas, irradiado hacia el flanco abdominal, región inguinal y genitales externos (testículo o labios mayores) del lado afecto. La historia familiar de CN y la baja ingestión de agua son factores etiopatogénicos que deben ser investigados, además las ocupaciones y estilos de vida sedentarios, los ambientes y meses de verano calurosos, sobre todo en Julio y Agosto, aumentan la posibilidad de formación de litiasis urinaria y aparición de CN. La litiasis más frecuente en nuestro medio fue la de oxalato cálcico mono y dihidratado.

Palabras clave: Cólico nefrítico. Epidemiología. Etiopatogenia. Diagnóstico. 
Summary.- OBJECTIVES: To investigate epidemiologic, etiopathogenic and clinical factors associated with emergency renal colic (RC).

METHODS ANDS RESULTS: We performed a prospective cross-sectional multicenter case-control study of 146 patients treated for $R C$ at emergency departments. Data collected included age, sex, localization/severity of pain, symptoms, personal/family medical history, urine analysis, etiopathogenic factors, chemical composition of the lithiasis, and x-ray studies. Comparative statistical analysis was performed using SPSS 12.2 soffware. RC was more frequent in men; maximum incidence was bet ween $37-50$ years for both sex, with $36.3 \%$ in men and $21.23 \%$ in women; $60.27 \%$ of patients rated pain as severe; 140 RC patients (95.89\%) had urologic antecedents vs. 15 (10.27\%) controls without $R C(p<0.001)$. The most frequent presentation (93.83\%) was sudden intense lumbar-abdominal or lumbar pain; $23.28 \%$ of RC patients had family history for urinary lithiasis vs. $6.16 \%$ controls ( $p<0,001)$. Most RC patients were seen during summer $(36.58 \%), 82 \%$ of $R C$ patients drank $<2 L$ of water daily vs. $18.49 \%$ in non-RC patients $(p<0.001)$. Hematuria was found in 132 (90.41\%) patients with RC vs. $17(11.64 \%)$ in those without ( $p<0.001)$. Lithiasis was observed by KUB x-ray in $42.10 \%$ of RC patients vs. $57.89 \%$ controls, most frequent calculi composition was calcium oxalate monohydrate and dehydrate $(61,2 \%)$.

CONCLUSIONS: The incidence of urinary lithiasis and $R C$ in our health care area shows a male predominance. The characteristic pain of RC is severe and appears suddenly. It starts in the back (lumbar region), below the ribs, radiating towards the groin and external genitals (testicles in man or major lips in woman) on the same side. Nausea and vomiting are frequent. Family history of urinary lithiasis and low water intake are risk factors that need to be investigated. Occupations associated with a sedentary life style or with a hot, dry workplace show a higher incidence of lithiasis. A hot, dry climate favours the formation of urinary lithiasis and the highest incidence of lithiasis is in the summer, during the months of July and August. The most frequent component of urolithiasis in our study, as well as in other studies, was calcium oxalate monohydrate and dihydrate.

Keywords: Renal colic. Epidemiology. Etiopathogenesis. Diagnosis.

\section{INTRODUCCIÓN}

En el Diccionario de la Lengua Española se define el término dolor como "sensación molesta y aflictiva de una parte del cuerpo por causa interior o exterior". La Asociación Internacional para el Estudio del Dolor nos ofrece una definición "más médica": "sensación física y emocional desagradable asociada a una lesión tisular potencial o real" (1).

El cólico nefrítico $(\mathrm{CN})$ es la presentación clínica más frecuente de la litiasis renal y una de las principales urgencias urológicas. Hasta un 10-20\% de los varones y un 3-5\% de las mujeres sufrirán al menos un episodio de $\mathrm{CN}$ durante su vida. En España la urolitiasis tiene una prevalencia del $4,2 \%$, con una mayor incidencia en la tercera y quinta décadas $y$ un claro predominio en varones $(4: 1)$. Origina un considerable sufrimiento en los pacientes y un elevado coste socioeconómico (2).

El CN, por la intensidad del dolor y el cotejo sintomático que lo acompaña, ocasiona un gran sufrimiento a los pacientes que lo padecen, los médicos, y en especial los Urólogos y Médicos de los Servicios de Urgencias, en numerosas ocasiones somos testigos directos del mismo; ante esta situación nuestro principal reto es el alivio lo más inmediato posible de dicho dolor, pero también es de gran importancia el conocimiento de las causas que lo produce. Con nuestro trabajo investigativo, realizado en el marco del Servicio de Urgencias, y con la colaboración en algunos aspectos de la consulta de Atención Primaria, Laboratorio de Análisis Clínicos y Departamento de Radiología, queremos profundizar en el estudio de esta patología y así propiciar un mejor conocimiento de los aspectos epidemiológicos, clínicos y etiopatogénicos de la misma.

De la terapéutica de esta afección se hablará en otra publicación, ya en fase de culminación, dedicada a la misma.

\section{OBJETIVOS}

\section{Generales}

Hacer un estudio sobre los factores epidemiológicos, etiopatogénicos, clínicos, de laboratorio y radiológicos, en los pacientes tratados por $\mathrm{CN}$ en el Servicio de Urgencias.

\section{Específicos}

Conocer la distribución de los pacientes afectos de $\mathrm{CN}$ por sexo y grupos etáreos.

Investigar los antecedentes patológicos personales (APP) de los pacientes con CN.

Estudiar el cuadro clínico del $\mathrm{CN}$ valorando: - La localización (derecho o izquierdo) y severidad del dolor de origen reno-ureteral en nuestros pacientes (leve, moderado, severo). 
- Las características clínicas del dolor de origen renoureteral en términos de:

a) Localización lumboabdominal e irradiación.

b) Síntomas acompañantes.

c) Intensidad.

Detectar la presencia de factores etiopatogénicos favorecedores de la formación de litiasis urinaria en nuestra casuística.

Valorar la presencia de factores hereditarios implicados en la etiopatogenia de la enfermedad litiásica, conociendo:

- Los antecedentes patológicos familiares (APF) de nuestros pacientes.

- Positividad o no del HLA B-27

Determinar la época del año de mayor prevalencia de $\mathrm{CN}$.

Investigar las alteraciones encontradas en la orina de los pacientes afectos, determinado el $\mathrm{pH}$ urinario, densidad urinaria, test de nitritos, leucocituria, hematuria, proteinuria, glucosuria, cetonuria.

Valorar los hallazgos radiológicos que podemos encontrar en un paciente con $\mathrm{CN}$, la capacidad de visualización de los cálculos mediante la radiografía simple de abdomen, su localización en el tracto urinario y aspecto radiográfico.

Conocer la composición química de los cálculos renales en nuestro medio.

Elaborar tablas y gráficos para mostrar los resultados obtenidos.

Hacer un análisis comparativo estadístico mediante el hallazgo del valor de $p$, para determinar si nuestros resultados son significativos.

\section{MATERIAL Y MÉTODO}

\section{Tipo de estudio y pacientes}

Se realizó un estudio clínico-epidemiológico, prospectivo, multicéntrico, transversal con un total de 146 pacientes $(n=146)$ que fueron tratados en el Servicio de Urgencias afectos de $\mathrm{CN}$, también se incluyeron un grupo de casos control $\sin C N(n=146)$, para hacer un análisis comparativo estadístico entre ambos grupos de pacientes sobre diferentes parámetros investigados.

\section{Recogida de datos}

Se revisaron las historias clínicas elaboradas en los diferentes servicios de urgencias (Modelo EAP54, del Servicio Canario de Salud) para conocer la edad, sexo, APP, APF, época del año en que sufrió el $\mathrm{CN}$, cuadro clínico, exámenes complementarios: urianálisis con Comburtest@ y en radiografía simple de abdomen. También se contó con la colaboración de diferentes consultas de Medicina Familiar en la investigación de ciertos parámetros.

\section{Características del dolor} lor referente a:

Se valoró las características clínicas del do-

a) Localización.

b) Irradiación

c) Intensidad: Nivel de percepción del paciente, de la intensidad del del dolor, utilizando como recursos la escala de categorías del dolor. En dicha escala hay cuatro categorías: ninguno, leve, moderado y severo. Dando el paciente una puntuación del 0 al 10.

Escala de categorías de dolor:

\begin{tabular}{|l|l|l|l|}
\hline Ninguno (0) & Leve (1-3) & Moderado (4-6) & Severo (7-10) \\
\hline
\end{tabular}

d) Percepción de la intensidad del dolor por parte de los facultativos en: leve, moderada o severa.

La clasificación de la intensidad del dolor de los facultativos fue la siguiente:

- Intensidad leve: Dolor soportable, paciente se presenta relativamente tranquilo, no ansiedad, deambula sin dificultad, describe pausadamente su cuadro clínico, puede haber alguna náusea, no hay palidez ni sudoración, otorga una puntuación al dolor del 1 al 3.

- Intensidad moderada: Dolor intenso, pero relativamente soportable, existe cierto grado de ansiedad e intranquilidad, puede haber náuseas 0 vómitos, describe de forma rápida su cuadro clínico, sudoración y palidez leves, puede adoptar cierta posición antiálgica al deambular, otorga una puntuación del 4 al 6.

- Intensidad severa: Dolor muy intenso, lo describe como una punzada en la región lumbar, ansioso, intranquilo, pálido, sudoroso, se queja constantemente y pide que "por favor le quiten el dolor", presenta náuseas y vómitos, a la deambulación adopta una posición antiálgica característica con el tronco semiflexionado y las manos apoyadas sobre el flanco o 
región lumbar dolorosos, otorga una puntuación del 7 al 10.

e) Síntomas acompañantes.

\section{Hallazgos al examen físico}

En todos los pacientes se procedió a la toma de constantes vitales que habitualmente se realizan en el Servicio de Urgencias (tensión arterial, frecuencia cardiaca, temperatura, glucemia capilar, saturación de oxígeno).

Se valoró la inspección, palpación, percusión y auscultación del abdomen, las fosas lumbares, exploración de los puntos pielorrenoureterales (PPRU) (dolorosos o no), teniendo presente los diagnósticos diferenciales del $\mathrm{CN}$.

\section{Factores etiopatogénicos implicados en la formación de litiasis urinaria}

A través de una encuesta, elaborada para ser rellenada en consulta, tanto por los pacientes afectos de $C N(n=146)$ como el grupo de casos control sin $C N(n=146)$, así como, realizando analíticas sanguíneas determinado el HLA B-27 de nuestros pacientes (con $\mathrm{CN} n=50$, sin $\mathrm{CN} n=50$ ) se realizó un estudio comparativo para valorar la presencia de factores epidemiológicos y etiopatogénicos favorecedores de la formación de litiasis urinaria en nuestra casuística, se investigó:

\section{Factores genéticos (intrínsecos):}

- Edad y sexo

- Herencia familiar: presencia de antecedentes de litiasis urinaria en familiares de primer grado (padre, madre, hermanos, abuelos, tios)

- HLA B-27 positivo (con $\mathrm{CN} n=50, \sin \mathrm{CN} n=50$ ).

II. Factores medioambientales (extrínsecos):

1. Factores climáticos y estacionales: Época del año en que sufrieron el CN (Primavera, Verano, Otoño, Invierno).

2. Ingesta hídrica $(<2$ litros de agua/día)

3. Factores dietéticos:

a) Consumo excesivo de productos lácteos

b) Dieta rica en purinas

c) Dieta vegetariana

d) Dieta rica en proteínas

e) Añade sal sin restricción a los alimentos f) Consumo frecuente de zumos de cítricos (>1 litro/día) (naranja, limón, tomate)

g) Consumo frecuente de zumos de cítricos (>1 litro/día) junto con productos lácteos

4. Tratamientos médicos:

a) Tratamiento con tiazidas

b) Litio

c) Teofilinas

5. Ocupación:

a) Trabajos sedentarios (oficinista, administrativo, amas de casa, asesores, licenciados)

b) Agricultores

c) Cocineros

6. Actividad física:

a) Realiza ejercicio físico:

-Regularmente

-Irregularmente

-Sedentario

7. Sometidos a eventos estresantes

\section{Alteraciones en el examen de orina}

Se realizó análisis de la orina a nuestros pacientes con $C N(n=146)$ y casos control sin $C N$ $(n=146)$, a través de la utilización de tiras reactivas (Comburtest $\circledast$ ), valorando los siguientes parámetros: $\mathrm{Ph}$, densidad, nitritos, leucocitos, hematíes, proteínas, glucosa, cuerpos cetónicos.

\section{Estudios radiológicos}

También se valoró la capacidad de visualización de los cálculos mediante la radiografía simple de abdomen, realizada a 76 pacientes. $(n=76)$.

Se analizó el aspecto radiológico de la litiasis, acorde a la densidad radiográfica observada:

- Radiopa

- Radiolúcida

- Mixta

Localización de la litiasis:

- Renal: Intraparénquimatosa, calicial o piélica

- Ureteral: Ureter superior, medio e inferior

- Vesical

- Uretral

\section{Composición química de la litiasis}

Se accedió a la base de datos de un Laboratorio de Análisis Clínicos, ubicado en nuestra Área de Salud. Se investigaron un total de 116 cálculos analizados en dicho laboratorio. Se utilizó el método 
de espectrometría infrarroja, mediante un Espectrómetro IMPACT 410 ISAZA®.

\section{Estudio estadístico}

Se realizó un análisis comparativo estadístico entre los 146 pacientes con $\mathrm{CN}$ y 146 casos control sin $\mathrm{CN}$, mediante el hallazgo de el valor de $p$, usando la prueba de Kruskal-Wallis, para un orden simple de clasificación, salvo las comparaciones de proporciones en gastritis y úlcera de estómago, que se realizó con la Prueba de Jonckeere-Tepstra para un doble orden de clasificación.

\section{Muestra de los resultados obtenidos}

Con todos la información y los datos recopilados se elaboraron tablas y gráficos, los que se muestran en el apartado correspondiente.

\section{Búsqueda de bibliografía}

La búsqueda de bibliográfica se efectuó a través de Internet visitando los principales portales médicos y páginas web de revistas, así como utilizando libros de texto y revistas de referencia en el tema estudiado.

\section{RESULTADOS Y DISCUSIÓN}

La prevalencia poblacional de la litiasis urinaria en España es del 4,16\% y la incidencia estimada es del $0,27 \%$, afectando al varón en un $4,5 \%$ y a la mujer en un $3,8 \%$, con un pico de máxima frecuencia entre los 30 y los 40 años de edad, según un estudio

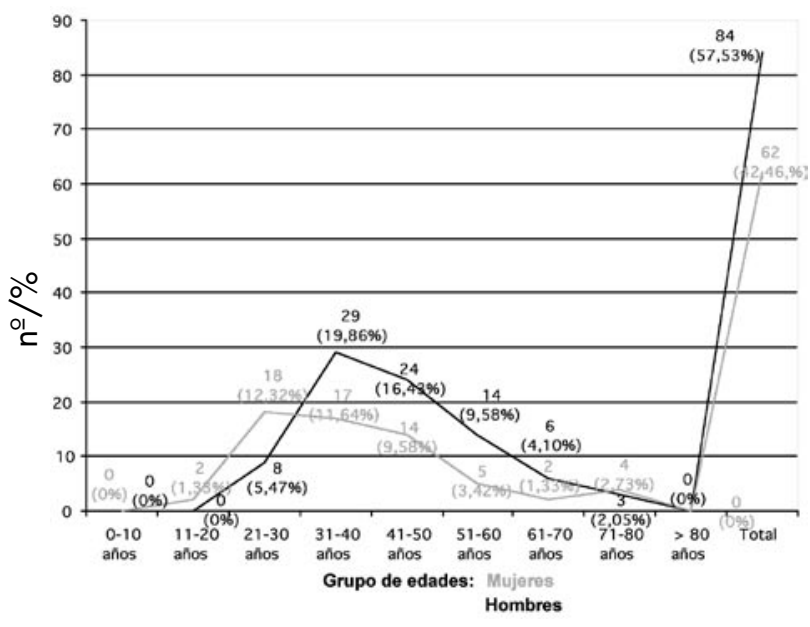

FIGURA 1. Distribución del CN por sexo y grupos etáreos. epidemiológico de la urolitiasis en España, realizado por el Grupo de Urolitiasis de la Asociación Española de Urología (3). Más recientemente, en un trabajo realizado con el objetivo de determinar la incidencia y la prevalencia actual de la urolitiasis en España, los autores concluyen que de entre 45 publicaciones de contenido epidemiológico sobre urolitiasis en España, se han publicado 16 trabajos que aportan datos originales sobre incidencia o prevalencia; la media obtenida de todos ellos, arroja una incidencia del $0,73 \%$ correspondiente a 325.079 nuevos casos por año; y una prevalencia de $5,06 \%$, correspondiente a 2.233.214 casos totales (4). Cuando observamos la distribución del $\mathrm{CN}$ por grupos etáreos en nuestra casuística, en ambos sexos, nuestros resultados coinciden con los datos anteriormente citados. En España hay un claro predominio en varones (4:1) (2), en nuestro trabajo un $57,53 \%$ de los pacientes fueron varones frente al $42,46 \%$ de mujeres (Figura 1 ).

Alapont y cols. (2001) (5), en un estudio realizado sobre epidemiología de la litiasis urinaria, detectan que la incidencia de la misma en su área de salud es elevada, siendo en 1998 de 2,66 por mil habitantes (156/58591), excluyendo la edad infantil (0 a 15 años), ellos destacan que la incidencia aumenta a 3,30 por mil habitantes adultos y año (156) 47328); la edad media es de 51,34 años con un intervalo de 13 a 91 años, la edad media en varones es menor que en mujeres $(50,93$ vs. 51,85$)$. El $67,2 \%$ de los pacientes tienen entre los 30 y los 70 años, siendo entre los 60 y los 69 años la década de máxima incidencia $(19,5 \%)$ con 173 casos. En nuestro estudio la década de mayor número de pacientes afectos de CN fue entre los 31-40 años, con un total de 46 pacientes $(31,50 \%)$, de estos 29 $(19,86 \%)$ fueron hombres y $17(11,64 \%)$ mujeres. En la edad infantil (menores de 15 años), los autores anteriormente mencionados, aprecian solamente un caso en toda la serie estudiada, mientras que nosotros no tratamos ningún paciente en edad infantil.

Respecto al sexo, en el estudio anteriormente citado (5), predominan los varones sobre las mujeres (495/392), con una relación hombre/mujer de 1,26, referido sólo a la edad adulta, la prevalencia fue de 3,94 por mil varones $(93 / 23596)$ y 2,65 por 1.000 mujeres (63/23732). Nosotros, del total de 146 pacientes tratados por $\mathrm{CN}, 84(57,53 \%)$ fueron hombres y 62 mujeres $(42,46 \%)$ (Figura 1).

En la Figura 2 mostramos la distribución del $\mathrm{CN}$ por la localización y severidad/intensidad del dolor. Con respecto a la localización, un $56,16 \%$ de $\mathrm{CN}$ fueron derechos frente al $43,83 \%$ izquierdos. Al hacer la valoración de la intensidad del dolor, se tuvo en cuenta los diferentes recursos para evaluar el do- 
lor, que ayudan a los pacientes a describir el dolor que sienten. La escala del dolor es un recurso que se usa comúnmente para describir la intensidad del dolor. Las escalas del dolor incluyen la escala de clasificación numérica, la escala análoga visual, la escala de categorías (aplicada por nosotros) y la escala de rostros de dolor (6). Un 60,27\% de nuestros pacientes dieron una puntuación de 7-10 por lo que lo catalogamos como intensidad severa, el 18,49\% dio una puntuación de $4-6$ (dolor moderado) y un $21,23 \%$ otorgaron una puntuación de 1-3 (dolor leve), nuestros hallazgos coinciden con lo que se plantea en la literatura revisada donde se cataloga al cólico renal como uno de los dolores más intensos que conoce el ser humano (1-4).

Desde el punto de vista de los APP (Tabla I), el $45,89 \%$ de nuestros pacientes con $C N(n=146)$ habían padecido cólicos nefríticos anteriores, en el grupo de casos control sin $\mathrm{CN}(\mathrm{n}=146)$ sólo el $1,36 \%$ padeció de estos $(p<0,001)$, en la literatura se dice que las tasas de recurrencia del $\mathrm{CN}$ son altas, del $35-50 \%$ a los 5 años (2). William y cols. (1963) (7) plantearon que se observa un porcentaje de recurrencias tan alto como un $50 \%$ a los 5 años, $60 \%$ a los 8 años y de hasta un $70 \%$ en plazos mayores de tiempo. En total 140 pacientes con CN $(95,89 \%)$ presentaron antecedentes de patología urológicas vs. $15(10,27 \%)$ en los $\sin C N(p<0,001)$.

Tratamos de $\mathrm{CN}$ a un paciente con sarcoidosis, enfermedad esta no muy frecuente, pero implicada en la patogenia de la litiasis urinaria, esta patología es la causa más común de nefrolitiasis hipercalcémica, entre las enfermedades granulomatosas, el granuloma sarcoideo produce 1, 25 -dihidroxivitamina D3, lo cual provoca un aumento en la absorción intestinal de calcio, hipercalcemia e hipercalciuria (8).

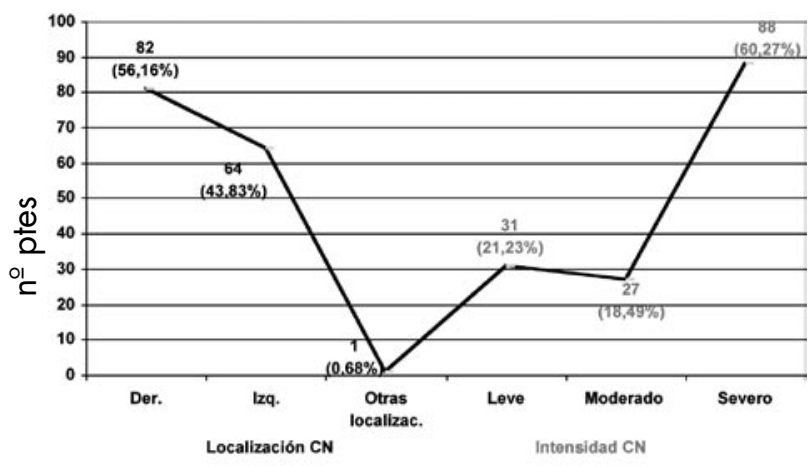

FIGURA 2. Distribución del CN por la localización y severidad del dolor.
En un estudio comparativo, con un total de 40 pacientes con litiasis de oxalato cálcico monohidrato, los autores, al analizar los factores etiológicos, concluyen que en estos pacientes se detectó un déficit de inhibidores de la cristalización y a una mayor presencia de nucleantes heterogéneos, materia orgánica inducida por enfermedades como la hipertensión arterial (HTA), que en nuestro estudio se observó en 60 pacientes con CN $(41,09 \%)$ y en $23 \sin \mathrm{SN}(15,75 \%)$ $(p<0,001)$, la hiperuricemia, hiperglucemia e hipercolesterolemia. Estos autores también detectan mayor exposición a agentes citotóxicos y elevada prevalencia de úlcera gastroduodenal; referente a esta última patología en nuestro trabajo 5 pacientes con $\mathrm{CN}$ $(3,42 \%)$ padecieron úlcera gástrica vs. 0 paciente en los $\sin C N(0 \%),\left(p=0,06 / 0,03^{\prime \prime}\right), 7$ pacientes con $\mathrm{CN}(4,45 \%)$ vs. 1 paciente sin $\mathrm{CN}(0,68 \%)$ padecieron gastritis $(p=0,07 / 0,02$ ") ("Se realizó con la Prueba de Jonckeere-Tepstra para un doble orden de clasificación) (9).

Alapont y cols. (2001) (5) al comparar la incidencia de patología asociada entre los pacientes litiásicos y su grupo de casos controles, no encontraron diferencias con significación estadística ( $p>0,05)$. Ellos detectan en los pacientes litiásicos 53 diabéticos vs. 3 en los casos controles, ulcus gástrico 119 vs. 11 , gota-hiperuricemia 90 vs. 10, patología biliar 56 vs. 6, artrosis 91 vs. 9, patologías respiratorias 64 vs. 8 , enfermedades cardíacas 56 vs. 5 , HTA 191 vs. 20 cefaleas 37 vs. 5 , otras 92 vs. 10, en total los litiásicos con enfermedades asociadas fueron 849 y en los casos control 87.

Desde el punto de vista del cuadro clínico (Tabla II), lo más característico es la presencia de un dolor abdominal o lumbar de inicio brusco unilateral, lancinante, de tipo cólico, irradiado a las ingles o los genitales, que no mejora con el reposo (2), aunque en algunos casos se presenta como dolor sordo intermitente localizado en fosa renal y/o fosa iliaca (5). En nuestra casuística fue más frecuente $(93,83 \%)$ la forma de presentación con dolor intenso, brusco, de localización lumboabdominal o lumbar, primando el dolor brusco lumboabdominal derecho $(42,46 \%)$ y en el $6,16 \%$ de los pacientes presentó un dolor brusco en diferentes localizaciones abdominales, lo que coincide con las observaciones de los diversos autores $(2,5)$. En el estudio de Alapont y cols. (2001) (5) el motivo de consulta más frecuente fue el dolor renoureteral tipo cólico $(54,78 \%)$, seguido a gran distancia por la microhematuria $(7,70 \%)$, la lumbalgia y las molestias miccionales.

El dolor puede irradiarse hacia la zona inguinal, si el cálculo esta en el trayecto lumbociático del uréter, mientras que, si la localización es más baja, 
TABLA I. ANÁLISIS COMPARATIVO SOBRE LAS PRINCIPALES PATOLOGÍAS ENCONTRADAS EN NUESTROS DOS GRUPOS DE PACIENTES, CON CN (N=146) Y SIN CN (N=146).

\begin{tabular}{|c|c|c|c|c|c|c|}
\hline \multirow[b]{2}{*}{ Patología } & \multicolumn{2}{|c|}{ Ptes con $C N(n=146)$} & \multicolumn{2}{|c|}{ Ptes $\sin C N(n=146)$} & \multirow{2}{*}{\multicolumn{2}{|c|}{ Valor de $p$}} \\
\hline & № & $\%$ & № & $\%$ & & \\
\hline Cólicos nefríticos anteriores & 67 & 45,89 & 2 & 1,36 & & .001 \\
\hline Cólico nefrítico del embarazo & 3 & 2,05 & 0 & 0 & & \\
\hline Expulsador de cálculos o "arenilla" & 14 & 9,58 & 1 & 0,68 & & .001 \\
\hline Poliquistosis renal & 1 & 0,68 & 0 & 0 & & \\
\hline Sarcoidosis & 1 & 0,68 & 0 & 0 & & \\
\hline Litiasis renal actual conocida & 13 & 8,90 & 1 & 0,68 & & 001 \\
\hline Ureterocele & 3 & 2,05 & 0 & 0 & & \\
\hline Riñón ectópico & 1 & 0,68 & 0 & 0 & & \\
\hline Nefrocalcinosis & 1 & 0,68 & 0 & 0 & & \\
\hline LEOC 1 reciente (expulsando restos) & 2 & 1,36 & 0 & 0 & & \\
\hline LEOC no reciente & 3 & 2,05 & 1 & 0,68 & & \\
\hline Obesidad & 48 & 32,87 & 21 & 14,38 & & 001 \\
\hline Urosepsis baja & 14 & 9,58 & 9 & 6,16 & & \\
\hline HTA2 & 60 & 41,09 & 23 & 15,75 & & 001 \\
\hline Diabetes Mellitus & 12 & 8,21 & 29 & 19,86 & & .006 \\
\hline Dislipemia & 11 & 7,53 & 25 & 17,12 & & \\
\hline Gastritis & 7 & 4,75 & 1 & 0,68 & $=0,07$ & $=0,02^{*}$ \\
\hline Ülcera de estómago & 5 & 3,42 & 0 & 0 & $=0,06$ & $=0,03^{*}$ \\
\hline Depresión & 5 & 3,42 & 5 & 3,42 & & \\
\hline Embarazo & 4 & 2,73 & 11 & 7,53 & & \\
\hline Urosepsis alta & 3 & 2,05 & 0 & 0 & & \\
\hline Asma & 3 & 2,05 & 11 & 7,74 & & \\
\hline HBP3 & 3 & 2,05 & 3 & 2,05 & & \\
\hline Hernia del hiato & 3 & 2,05 & 6 & 4,10 & & \\
\hline Diverticulosis & 2 & 1,36 & 1 & 0,68 & & \\
\hline Cardiopatía isquémica & 2 & 1,36 & 8 & 5,47 & & \\
\hline Catéter doble J por hidronefrosis & 1 & 0,68 & 0 & 0 & & \\
\hline Hipotiroidismo & 1 & 0,68 & 3 & 2,05 & & 006 \\
\hline Hiperuricemia & 13 & 8,90 & 2 & 1,36 & & \\
\hline Hipercalciuria & 2 & 1,36 & 0 & 0 & & \\
\hline Osteoporosis & 8 & 5,47 & 13 & 8,90 & & \\
\hline Hiperparatiroidismo & 1 & 0,68 & 0 & 0 & & \\
\hline Apendicectomizado & 4 & 3,17 & 7 & 4,79 & & \\
\hline Enfermedad inflamatoria intestinal & 2 & 1,36 & 0 & 0 & & 001 \\
\hline Antecedentes de patología urológica & 140 & 95,89 & 15 & 10,27 & & \\
\hline Neoplasias & 0 & 0 & 3 & 2,05 & & \\
\hline Tetraplejia/Paraplejia/Inmovilizado & 1 & 0,68 & 1 & 0,68 & & \\
\hline
\end{tabular}

1 LEOC: Litotricia Extracolpórea por Ondas de Choque 2 HTA: Hipertensión arterial

3 HBP: Hiperplasia Benigna de la Próstata. *Se realizó con la Prueba de Jonckeere-Tepstra para un doble orden de clasificación. 
TABLA II. CUADRO CLÍNICO DEL CN.

\begin{tabular}{|c|c|c|c|}
\hline SÍNTOMAS & № & & $\%$ \\
\hline \multicolumn{4}{|c|}{ Forma de comienzo y localización del dolor } \\
\hline Dolor brusco LA1 derecho. & 62 & & 42,46 \\
\hline Dolor brusco LA izquierdo & 54 & & 36,98 \\
\hline Dolor sordo, intermitente LA derecho & 3 & & 2,05 \\
\hline Dolor sordo, intermitente LA izquierdo & 2 & & 1,36 \\
\hline Dolor brusco lumbar derecho & 7 & & 4,79 \\
\hline Dolor brusco lumbar izquierdo & 5 & & 3,42 \\
\hline Dolor sordo lumbar derecho & 3 & & 2,05 \\
\hline Dolor sordo lumbar izquierdo & 1 & 0,68 & Total dolor lumbar: 137 (93,83\%) \\
\hline Dolor en flanco abdominal derecho & 3 & & 2,05 \\
\hline Dolor en flanco abdominal izquierdo & 2 & & 1,36 \\
\hline Dolor en fosa iliaca derecha & 2 & & 1,36 \\
\hline Dolor en fosa iliaca izquierda & 1 & & 0,68 \\
\hline Dolor en bajo vientre & 1 & 0,68 & Total dolor abdominal: $9 \quad(6,16 \%)$ \\
\hline \multicolumn{4}{|l|}{ Irradiación del dolor } \\
\hline Genitales (escroto, labios >) & 25 & & 17,12 \\
\hline Cara interna del muslo & 12 & & 8,21 \\
\hline Cara externa del muslo & 1 & & 0,68 \\
\hline Región inguinal & 13 & & 8,90 \\
\hline Flanco abdominal y fosa iliaca & 5 & & 3,42 \\
\hline Bajo vientre & 4 & 2,73 & Total dolor irradiado: $60 \quad(41,09 \%)$ \\
\hline \multicolumn{4}{|l|}{ Síntomas acompañantes } \\
\hline Hematuria macroscópica & 19 & & 13,01 \\
\hline Disuria, polaquiuria, tenesmo vesical. & 27 & & 17,80 \\
\hline Síntomas vegetativos (palidez, sudoración) & 8 & & 5,47 \\
\hline Nauseas & 65 & & 44,52 \\
\hline Nauseas y vómitos & 24 & & 16,43 \\
\hline Pujos y tenesmo rectal & 13 & & 8,90 \\
\hline Sequedad bucal & 1 & & 0,68 \\
\hline Agitación, inquietud & 22 & & 15,06 \\
\hline Fiebre & 5 & & 3,42 \\
\hline Otros (fatiga, escalofríos, llorando) & 4 & 2,73 & Total síntomas acompañantes: 166 \\
\hline \multicolumn{4}{|l|}{ EXÁMEN FíSICO } \\
\hline \multicolumn{4}{|l|}{ Inspección } \\
\hline Paciente ansioso, intranquilo, se queja. & 22 & & 15,06 \\
\hline \multicolumn{4}{|l|}{ Palpación } \\
\hline Dolor en FLD2 & 75 & & 51,36 \\
\hline Dolor en la FLI3 & 62 & & 42,46 \\
\hline PPRUD4 dolorosos & 80 & & 54,79 \\
\hline PPRUI5 dolorosos & 65 & & 44,52 \\
\hline Dolor en flanco abdominal derecho & 9 & & 6,16 \\
\hline Dolor en flanco abdominal izquierdo & 7 & & 4,79 \\
\hline Dolor en el bajo vientre & 12 & & 8,21 \\
\hline Dolor en el HD6 & 1 & & 0,68 \\
\hline Dolor en fosas iliacas & 6 & & 4,10 \\
\hline Percusión: Puño percusión positiva & 137 & & 93,83 \\
\hline \multicolumn{4}{|l|}{ Auscultación: } \\
\hline Ruidos hidroaereos algo $\downarrow$ & 5 & & 3,42 \\
\hline Soplo abdominal o lumbar & 0 & & 0 \\
\hline
\end{tabular}

'LA: Lumboabdominal. ${ }^{2} F L D$ : fosa lumbar der. ${ }^{3} F L I$ : Fosa lumbar izq. ${ }^{4}$ PPRUD: puntos pielorrenoureterales der. ${ }^{5}$ PPRUI: puntos pielorrenoureterales izq. ${ }^{6} \mathrm{HD}$ : hipocondrio derecho. 
el dolor se irradia a la cara interna de los muslos y genitales, en nuestro estudio el dolor se irradió en el $41,09 \%$ de los pacientes, siendo la irradiación más frecuente a genitales (escroto/ labios mayores) con un $17,12 \%$, seguido de la irradiación a región inguinal con el $8,90 \%$ y cara interna del muslo en el $8,21 \%(10)$.

Con respecto a los síntomas acompañantes, sabemos que el $\mathrm{CN}$ es un cotejo sintomático que tiene como síntoma cardinal del dolor de diversas características en cuanto a intensidad y localización, a veces con una sintomatología muy florida y característica, puede haber asociado síntomas vegetativos como son: nauseas, el más frecuente, visto en el $44,52 \%$ de nuestros pacientes, nauseas acompañado de vómitos $(16,43 \%)$, también podemos observar agitacióninquietud $(15,06 \%)$, palidez, sudoración $(5,47 \%)$ e ileo paralítico, la presencia de estos síntomas asociados al cuadro doloroso en el $\mathrm{CN}$ está recogida en la literatura $(2,10)$. También se plantea que se puede acompañar de hematuria macro y microscópica, por la acción erosiva directa de la litiasis sobre el urotelio, vista en el $13,01 \%$ y $90,41 \%$ de nuestra casuística respectivamente, y que pueden aparecer síntomas urinarios como polaquiuria y tenesmo vesical, sobre todo en las litiasis localizadas en los tramos inferiores y porción yuxtavesical del uréter, lo que se observó en el $17,80 \%$ de nuestros pacientes $(2,5,10)$.

Uno de los aspectos más importantes de la enfermedad litiásica son los factores etiopatogénicos y epidemiológicos favorecedores de la formación de cálculos en la vía urinaria (Tabla III), y estos, al ser tan numerosos y complejos, son objeto de constante investigación por parte de la comunidad médica interesada en este tema. Existen factores intrínsecos y extrínsecos. Dentro de los intrínsecos tenemos: los genéticos, alrededor de un $25 \%$ de los pacientes con cálculos renales tienen una historia familiar de dicha patología (11), en nuestra casuística un $23,28 \%$ de pacientes con $\mathrm{CN}(\mathrm{n}=146)$ refirieron presentar antecedentes familiares de enfermedad litiásica, en los pacientes control sin $\mathrm{CN}(\mathrm{n}=146)$ se detectó este antecedente en el 6,16\% ( $p<0,001)$, Alapont y cols. (2001) (5) detectan antecedentes de litiasis urinaria familiar en 181 pacientes $(20,41 \%)$, siendo el padre el parentesco más afectado con un $12,18 \%$ seguido de la madre $(7,72 \%)$, pero no hallan diferencias estadísticamente significativas con el grupo control.

Se han realizado otros trabajos para demostrar el papel de la herencia en la etiopatogenia de la enfermedad litiásica, y entre estos queremos destacar un análisis estadístico realizado para evaluar el antecedente familiar de litiasis, en el mismo los autores emplean el método de $\chi$ cuadrada y la prueba de $\dagger$ de Student que se utilizó para establecer comparaciones, ellos observan que el $44 \%$ de los sujetos con litiasis definitiva (LD) y $34 \%$ de los sujetos con litiasis probable (LP) tuvieron historia familiar de litiasis, comparado con $28,1 \%$ de los sujetos sin litiasis (SL) $(\mathrm{p}<0.0001), \mathrm{RM}$ (Razón de momios cruda) $=2,8$, IC $95 \%=2,2-3,4$ y $\mathrm{p}<0.0001, \mathrm{RM}=1,8, \mathrm{IC} 95 \%=1,4-$ 2,4 , respectivamente. Cuando analizan la prevalencia del antecedente familiar único en el padre, la madre o hermanos de los sujetos con LD, comparado con los sujetos $\mathrm{SL}$, esta fue mayor en los hermanos $(p<0.0001), R M=2.6, I C \quad 95 \%=1.83-3.80)$. Se observó un patrón similar en el grupo LP (12).

Otro factor relacionado con la herencia investigado por nosotros fue la positividad o no del HLA B-27, se realizó a 50 pacientes con $C N$ y 50 sin $\mathrm{CN}$, no encontrándose ningún paciente HLA-B-27 positivo en esta casuística, sin embargo, hay autores que plantean que se ha demostrado un aumento de la frecuencia de este antígeno en los pacientes litiásicos (13). La edad y el sexo, catalogados como factores intrínsecos, son analizados en la Figura 1.

Dentro de los factores extrínsecos tenemos a la geografía, planteándose que la prevalencia de cálculos urinarios es más alta en aquellos que viven en áreas montañosas, desérticas o tropicales, condiciones estas que se ajustan en cierta medida a las características geográficas y climatológicas de $\mathrm{Ca}$ narias (14). Referente a los factores climáticos y estacionales, también incluidos dentro de los factores extrínsecos, Prince y cols. (1956) (15) encontraron que la incidencia de cálculos urinarios era más alta durante los meses de verano (Julio, Agosto y Septiembre). Alapont y cols. (2001) (5) observan una mayor incidencia de inicio de la clínica en verano durante los meses de Julio y Agosto con un 13,53\% (120 casos) y un $11,05 \%$ (98 casos) respectivamente y la menor en invierno, meses de Diciembre y Enero con un 4,28 (38 casos) y un $6,09 \%$ (54 casos) respectivamente, según este estudio, la prevalencia en verano ( 15 de Junio a 15 de Septiembre) fue de 292 casos (33\%), en otoño (15 de Septiembre al 15 de Diciembre) de 231 casos y un $26 \%$ (IC 95\%), en invierno (15 de Diciembre a 15 de Marzo) de 169 casos (19\%) y en primavera (15 de Marzo a 15 de Junio) de 195 casos (22\%), la diferencia fue estadísticamente significativa entre los meses de verano y los meses de invierno y primavera con una $p<0,0013$ y una $p<0,0095$ respectivamente. La diferencia entre los meses de verano y los meses de otoño no fue estadísticamente significativa $(p<0,086)$, como podemos observar (Tabla III) nuestros resultados coinciden con los anteriores, comprobándose que en nuestro estudio el número de pacientes con cólicos nefríticos fue mayor durante los meses de verano (Julio, Agosto y 
TABLA III. ESTUDIO COMPARATIVO PARA VALORAR LA PRESENCIA DE FACTORES ETIOPATOGÉNICOS FAVORECEDORES DE LA FORMACIÓN DE LITIASIS URINARIA.

\begin{tabular}{|c|c|c|c|c|c|}
\hline \multirow{2}{*}{\begin{tabular}{|ll} 
& Pacientes \\
Factores & \\
\end{tabular}} & \multicolumn{2}{|c|}{ Ptes con $\mathrm{CN}^{1}(\mathrm{n}=146)$} & \multicolumn{2}{|c|}{ Ptes $\sin C N(n=146)$} & \multirow[t]{2}{*}{ Valor de $p$} \\
\hline & № & $\%$ & № & $\%$ & \\
\hline I. Factores genéticos: & & & & & \\
\hline 1. Herencia familiar ${ }^{2}$ & 34 & 23,28 & 9 & 6,16 & $<0,001$ \\
\hline 2.HLA $B-27^{3}$ positivo (con $\mathrm{CN} n=50, \sin \mathrm{CN} n=50$ ) & 0 & 0 & 0 & 0 & \\
\hline \multicolumn{6}{|l|}{ II. Factores medioambientales (extrínsecos) } \\
\hline \multicolumn{6}{|l|}{ 1. Factores climáticos y estacionales: } \\
\hline \multicolumn{6}{|l|}{ Época del año en que sufrieron el CN: } \\
\hline a) Enero-Febrero-Marzo & 32 & 21,91 & & & \\
\hline b) Abril- Mayo-Junio & 47 & 32,19 & & & \\
\hline c) Julio- Agosto- Septiembre & 54 & 36,98 & & & \\
\hline d) Octubre-Noviembre-Diciembre & 13 & 8,90 & & & \\
\hline 2. Ingesta $<2$ litros de agua/día & 118 & 80,82 & 27 & 18,49 & $<0,001$ \\
\hline \multicolumn{6}{|l|}{ 3. Dieta } \\
\hline a) Consumo excesivo de productos lácteos & 51 & 34,93 & 6 & 4,02 & $<0,001$ \\
\hline b) Dieta rica en purinas & 42 & 28,76 & 14 & 9,58 & $<0,001$ \\
\hline c) Dieta vegetariana & 14 & 9,58 & 3 & 2,05 & \\
\hline d) Dieta rica en proteínas & 89 & 60,95 & 26 & 17,80 & $<0,001$ \\
\hline e) Añade sal sin restricción a los alimentos & 127 & 86,98 & 59 & 40,41 & $<0,001$ \\
\hline $\begin{array}{l}\text { f) Consumo frecuente de zumos de cítricos (>1 } \\
\text { litro/día) (naranja, limón, tomate) * }\end{array}$ & 115 & 78,76 & 34 & 23,28 & $<0,001$ \\
\hline $\begin{array}{l}\text { g) Consumo frecuente de zumos de cítricos (>1 } \\
\text { litro/día) junto con productos lácteos }\end{array}$ & 122 & 83,56 & 19 & 13,01 & $<0,001$ \\
\hline \multicolumn{6}{|l|}{ 4. Tratamientos médicos } \\
\hline a)Tratamiento con tiazidas (Campbell T-4, p3561) & 18 & 12,32 & 12 & 8,21 & \\
\hline b)Litio & 0 & 0 & 1 & 0,68 & \\
\hline c)Teofilinas & 1 & 0,68 & 0 & 0 & \\
\hline \multicolumn{6}{|l|}{ 5. Ocupación: } \\
\hline $\begin{array}{l}\text { a) Trabajos sedentarios (oficinista, administrativo, } \\
\text { contables, asesores, licenciados) }\end{array}$ & 53 & 36,30 & 12 & 8,21 & $<0,001$ \\
\hline b) Agricultores & 25 & 17,12 & 18 & 12,32 & \\
\hline c) Cocineros & 9 & 6,16 & 6 & 4,10 & \\
\hline \multicolumn{6}{|l|}{ 6. Actividad física } \\
\hline \multicolumn{6}{|l|}{ a) Realiza ejercicio físico: } \\
\hline -Regularmente & 21 & 14,38 & 25 & 17,12 & \\
\hline -Irregularmente & 9 & 6,16 & 12 & 8,21 & \\
\hline b)Sedentario & 116 & 79,45 & 109 & 74,65 & \\
\hline 7. Sometidos a eventos estresantes & 89 & 60,95 & 21 & 14,38 & $<0,001$ \\
\hline
\end{tabular}

'CN: Cólico Nefrítico ${ }^{2}$ Antecedentes familiares de enfermedad litiásica ${ }^{3} \mathrm{HLA}$ B-27 se realizó a 50 pacientes con CN $(n=50)$ y a 50 pacientes $\sin C N(n=50)$

4 Parámetros no valorables 
Septiembre) con 54 casos $(36,98 \%)$ y menor durante los meses de otoño-invierno (Octubre, Noviembre, Diciembre) con 13 casos $(8,90 \%)$. Otro de los factores extrínsecos fue la ingesta de agua, Finlayson en 1974 (16) en un estudio realizado para determinar la eficacia de la ingestión forzada de agua como método de prevención de la recurrencia de la enfermedad litiásica, concluyó que el efecto dilucional de la diuresis acuosa pesa más que los cambios en la actividad iónica y, por lo tanto, ayuda a evitar la formación de cálculos $(9,16)$, como podemos apreciar en la Tabla III un $80,82 \%$ de nuestros pacientes atendidos por $\mathrm{CN}(\mathrm{n}=146)$ reconoció ingerir menos de 2 litros de agua al día, lo que, comparado con el grupo de casos control sin CN ( $n=146)$, en los que sólo el $18,49 \%$ reconoció ingerir menos de esta cantidad de agua al día, resultó ser estadísticamente significativo $(p<0,001)$.

Otros factores extrínsecos como son la dieta, donde se menciona al posible consumo excesivo de productos lácteos (leche, queso, yogourt, helados) reconocido por un $34,93 \%$ de nuestros pacientes con $C N(n=146)$ y el $4,02 \%$ de los $\sin C N(n=146)$ $(p<0,001)$, una dieta rica en purinas (carnes rojas, vísceras, pescados azules, marisco, quesos curados - fermentados, embutidos, carnes de caza, cacao, nueces, lentejas), detectado en un $28,76 \%$ de nuestros pacientes con $\mathrm{CN}$ y en el $9,58 \%$ de los pacientes control sin $\mathrm{CN}(\mathrm{n}=146)(\mathrm{p}<0,001)$, los efectos de estas dietas sobre la litogénesis han sido mencionado por diversos autores (17), también referente a la dieta el 9,58\% de los pacientes con CN ( $n=146)$ la dieta era predominantemente vegetariana vs. el 2,05\% de los casos control sin $C N(n=146)$, lo que no fue estadísticamente significativo, sin embargo se plantea que una dieta vegetariana produce una menor excresión de calcio, ácido oxálico y ácido úrico en la orina, con lo que se reduce la incidencia de cálculos urinarios y la tendencia a la recidiva. Debe propugnarse el consumo de vegetales con fibra y limitar el consumo de proteínas de origen animal, carne o pescado a unos $100 \mathrm{grs}$ diarios (18).

Referente a la ocupación el mayor número de pacientes que sufrieron $C N(n=146)$ se observó en aquellos que realizaron trabajos sedentarios como son: oficinistas, administrativos, contables, asesores (con CN ( $n=146)$ : $36,30 \%$, sin CN ( $n=146): 8,21 \%$, $\mathrm{p}<0,001)$, seguido de los agricultores $(17,12 \%)$ y los cocineros $(6,16 \%)(15)$, estos hallazgos coinciden con los de Alapont y cols. (2001) (5) los que plantean que por lo que respecta a la profesión se evidencia una mayor incidencia en personas sedentarias tales como pensionistas $(22 \%)$ y amas de casaempleadas de hogar $(28 \%)$ seguidos de agricultura e industria con un 13 y un $10 \%$ respectivamente. Otro de los factores extrínsecos investigados fue el estrés, donde un $60,95 \%$ de pacientes con $\mathrm{CN}(\mathrm{n}=146)$ refirió padecer eventos estresantes con frecuencia y en el grupo de casos controles se detectó esta factor en un $14,38 \%(p<0,001)$, se ha invocado también el efecto del estrés como factor de riesgo en la litiasis oxalocálcica, lo que explicaría la mayor incidencia de cálculos en hipertensos (en nuestro estudio HTA: pacientes con $\mathrm{CN}$ : $41,09 \%$ vs. $\sin \mathrm{CN} 15,75 \%$, $p<0,001)(19)$.

El tratamiento con tiazidas, también se encuentra entre los factores etiopatogénicos de la litiasis renal, estos diuréticos pueden causar hipercalcemia al incrementar la reabsorción tubular proximal de calcio reduciendo el volumen plasmático y mejorando la sensibilidad de los tejidos blandos a la PTH. En algunos pacientes la hipercalcemia inducida por tiazidas lleva el desenmascaramiento de un sutil hiperparatiroidismo primario (20), en nuestra casuística observamos un mayor número de pacientes con $\mathrm{CN}$ en tratamiento con tiazidas $18(12,32 \%)$ vs. 12 $(8,21 \%)$ en el grupo de controles sin $\mathrm{CN}, \mathrm{y}$ aunque estos datos no resultaron ser estadísticamente significativos, los queremos mencionar. De forma similar el litio puede inducir una condición que imita al hiperparatiroidismo primario, hay autores que han detectado que el calcio iónico sérico estaba aumentado en pacientes tratados con litio sin ningún cambio inicial en los niveles de PTH (21).

Analizando los resultados obtenidos en el urianálisis con Comburtest $\circledR$ (Tabla IV) vemos que en un $9,59 \%$ de los análisis realizados no se detectó microhematuria, se plantea que la presencia de microhematuria apoya el diagnóstico, si bien esta puede estar ausente en hasta el 15\% de los cólicos (2). Kobayashi y cols. (2003) (22) en un estudio realizado para determinar el impacto que tiene la fecha de la detección de hematuria mediante tiras reactivas - la ausencia de la misma durante la crisis aguda renoureteral, llegan a la conclusión de que la hematuria esta ausente en una significada proporción de pacientes con crisis renoureteral aguda.

La presencia de leucocitos $(18,49 \%)$, es uno de los hallazgos del urianálisis con Comburtes $₫$ que podemos observar en los pacientes que padecen $\mathrm{CN}$, sobre todo en aquellos afectos de Infección del Tracto Urinario (ITU), tanto alta $(2,05 \%)$ como baja $(9,58 \%)$. Es sabido que existe una gran relación clínica entre litiasis e infección urinaria; esta relación es de tipo multifactorial y compleja: papel obstructivo del cálculo que favorece la infección: obstrucción litiásica+infección= nefritis instersticial por reflujo pielocanalicular-pionefrosis-reflujo pielovenoso y pielolinfático-bacteriemia-shock séptico, además pueden 
ocurrir otros procesos como perinefritis, abscesos perinéfricos, pielonefritis enfisematosa (sobre todo en diabéticos e inmunocomprometidos), pielonefritis xantogranulomatosa y a la inversa, infección urinaria por gérmenes ureasa positivos que favorecen la litogénesis. Otras complicaciones que pueden aparecer, aunque tardíamente, son la pielonefritis crónica, el carcinoma epidermoide (litiasis+infección+obstrucci ón=respuesta anormal del urotelio-leucoplasia y colesteatoma-metaplasia escamosa-carcinoma epidermoide (23).

Con respecto a la determinación de la densidad urinaria (mediante el Comburtest $\circledast$ ) queremos primeramente destacar que esta varía entre 1001. 1035, suele reflejar el estado de hidratación del paciente, puede estar afectada por una función renal anormal y por la cantidad de material disuelto en la orina; una densidad de < de 1008 se considera diluida y una $>$ de 1020 se considera concentrada (24). En nuestro estudio detectamos que un total de 117 pacientes afectos de $\mathrm{CN}(\mathrm{n}=146)(80,13 \%)$, presentaron densidad urinaria entre 1020 a 1030, lo que comparado con los 43 pacientes $(29,45 \%)$ de el grupo de casos control $\sin \mathrm{CN}(\mathrm{n}=146)$, donde se detectó estas cifras de densidad urinaria, resultarían ser diferencias estadísticamente significativas $(p<0,001)$ por lo que podemos confirmar la presencia de una orina concentrada en la enfermedad litiásica.

Dentro de los factores de riesgo metabólico de la litiasis cálcica (75\% de los cálculos) tenemos las alteraciones del pH urinario, en un estudio con un total de 500 pacientes realizado en el "Hospital

TABLA IV. RESULTADOS DEL URIANÁLISIS (COMBURTEST®).

\begin{tabular}{|c|c|c|c|c|c|}
\hline \multirow[b]{2}{*}{ Parámetro urinario } & \multicolumn{2}{|c|}{ Ptes con $C N(n=146)$} & \multicolumn{2}{|c|}{ Ptes $\sin C N(n=146)$} & \multirow[t]{2}{*}{ Valor de $p$} \\
\hline & № & $\%$ & № & $\%$ & \\
\hline Hematíes negativo & 14 & 9,58 & 129 & 88,35 & \\
\hline Hematíes & 132 & 90,41 & 17 & 11,64 & $<0,001$ \\
\hline Leucocitos & 27 & 18,49 & 32 & 21,91 & \\
\hline Proteinas & 20 & 13,69 & 5 & 3,42 & $=0,003$ \\
\hline Nitritos & 6 & 4,10 & 3 & 2,05 & \\
\hline Glucosa & 9 & 6,16 & 8 & 5,47 & \\
\hline Cuerpos cetónicos & 9 & 6,16 & 1 & 0,68 & \\
\hline Urobilinógeno & 5 & 3,42 & 6 & 4,10 & \\
\hline \multicolumn{6}{|l|}{ Densidad urinaria } \\
\hline $1000-1015$ & 29 & 19,86 & 103 & 70,54 & \\
\hline $1020-1030$ & 117 & 80,13 & 43 & 29,45 & $<0,001$ \\
\hline \multicolumn{6}{|l|}{$\mathrm{pH}$ urinario } \\
\hline 5 (ácido) & 72 & 49,31 & 47 & 32,19 & $=0,002$ \\
\hline 6 & 33 & 22,60 & 35 & 23,97 & \\
\hline 7 (alcalino) & 35 & 23,97 & 42 & 28,76 & \\
\hline 8 (alcalino) & 6 & 4,10 & 22 & 15,06 & \\
\hline Total pH alcalino & 41 & 28,0 & 64 & 43,83 & $=0,007$ \\
\hline Comburtest $₫$ negativo & 3 & 2,05 & 100 & 68,49 & $<0,001$ \\
\hline
\end{tabular}


Juan Canalejo" de A Coruña (25), Lancina Martín (1999) detectó, de forma aislada: $\mathrm{pH}$ urinario ácido en el $8 \%$ de los pacientes y $\mathrm{pH}$ urinario alcalino en el $3 \%$, asociado encontró pH urinario ácido en un $15 \%$ y $\mathrm{pH}$ alcalino en el $6 \%$ de los pacientes; un $13 \%$ de cálculos pueden ser de ácido úrico, secundarios a hiperuricosuria y $\mathrm{pH}$ urinario ácido. Como podemos observar en la Tabla IV un 49,31\% de nuestros pacientes presentaba $\mathrm{pH}$ urinario ácido y el $28,08 \%$ presentó pH alcalino. Dentro de los factores de riesgo que pueden participar en la litogénesis de la litiasis úrica tenemos el $\mathrm{pH}$ urinario persistentemente ácido, por debajo de 6 (17).

La baja ingesta de líquidos, reconocida en un $80,82 \%$ de nuestros pacientes con $C N(n=146)$, provocaría estados de deshidratación con aumento de la densidad urinaria, lo que aumentaría el riesgo de formación de litiasis (24).

El Comburtest $₫$ fue negativo en el $2,05 \%$ de los pacientes con $\mathrm{CN}(\mathrm{n}=146)$ lo que corrobora la afirmación de que "un análisis de orina normal no excluye un $\mathrm{CN}^{\prime \prime}$ (23).

TABLA V. LOCALIZACIÓN DEL CÁLCULO EN EL RX ABDOMEN SIMPLE (N=32).

\begin{tabular}{|l|c|c|}
\hline Localización & № pacientes & $\%$ \\
\hline Intraparenquimatosa & 0 & 0 \\
\hline Cálices/pelvis & 5 & 15,62 \\
\hline Uréter: & 6 & 18,75 \\
Superior & 7 & 21,87 \\
Inferior & 12 & 37,5 \\
\hline Total cálculos ureterales & 25 & 78,12 \\
\hline Vejiga & 1 & 3,12 \\
\hline Uretra & 0 & 0 \\
\hline Otras localizaciones & 1 & 3,12 \\
\hline Total & 32 & 100 \\
\hline
\end{tabular}

*Riñón derecho ectópico, localizado en pelvis.

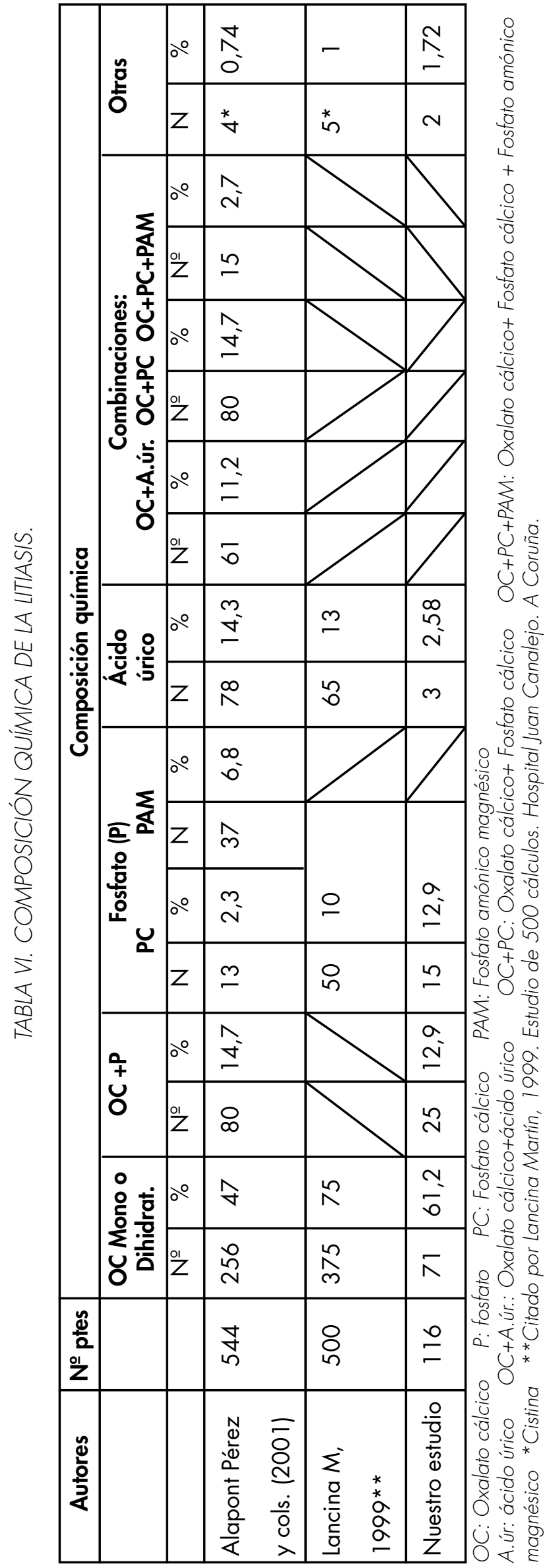


En la Figura 3 mostramos la capacidad de visualización de los cálculos mediante la radiografía simple de abdomen, este estudio radiológico lo realizamos en un total de 76 pacientes $(n=76)$, los que representan el $52,05 \%$ del total de la casuística $(n=146)$, como podemos observar se visualizó imagen sugestiva de litiasis en 32 pacientes, que representan el $42,10 \%$, de estos 29 fueron litiasis radiopacas $(38,15 \%), 3$ litiasis radiolúcidas $(3,94 \%)$. La radiografía abdominal simple puede ofrecer la visualización de sombras hiperdensas a cualquier nivel del tracto urinario. Su inexistencia no invalida el diagnóstico, ya que, la radioopacidad del cálculo depende de su contenido en sales cálcicas, lo que ocurre con los coágulos, cálculos de cistina y ácido úrico, que no presentan dichas sales, siendo radiolúcidos (26).

Uno de los problemas fundamentales en la identificación de la imagen litiásica surge cuando esta está localizada en el tercio distal del uréter, donde a veces resulta difícil diferenciarlas de otras densidades como los flebolitos, que con frecuencia se observan en el área de la pelvis, siendo redondos, mientras que los cálculos ureterales dan una imagen que a menudo se parece a la "raíz de un diente" (recogido en la literatura anglosajona como: "the tooth-root sign"), siendo de gran utilidad la clínica del paciente para la posible localización de la litiasis (27).

En un estudio de la Clínica Mayo, la capacidad de predecir la composición de los cálculos a partir de las radiografías simples fue de aproximadamente el $40 \%$, aun cuando las placas fueron leídas por un panel de expertos en urolitiasis, como podemos observar en nuestra casuística visualizamos litia-

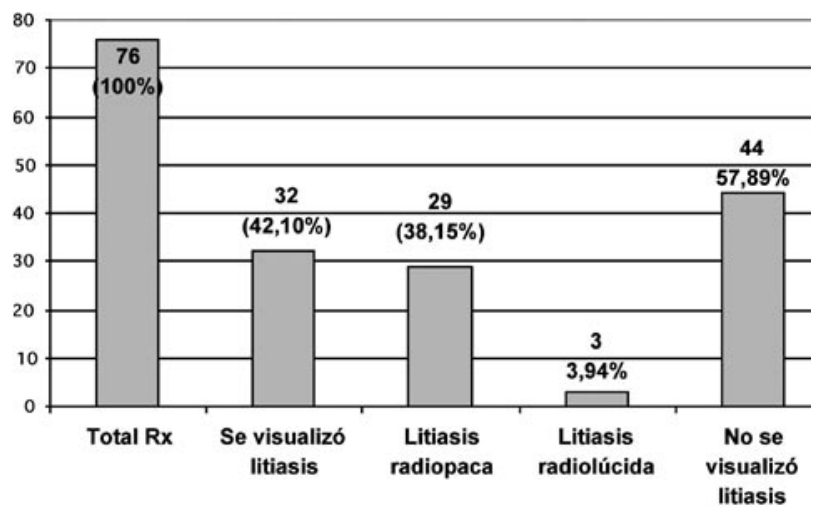

FIGURA 3. Capacidad de visualización de los cálculos mediante la radiografía simple de abdomen $(n=76)$. sis radiopacas en el $38,15 \%$ y litiasis radiolúcidas en el $3,94 \%(28)$.

Alapont y cols. (2001) (5) cuando analizan los métodos diagnósticos, la localización, el tipo de cálculo y el tratamiento efectuados, realizan $\mathrm{Rx}$ de abdomen simple a sus 887 pacientes (100\%), además ecografía, urografía intravenosa (UIV) y TAC, a un número determinado de los mismos. Estos últimos métodos de diagnóstico fueron imposible de ser utilizados y estudiados por nosotros, salvo en un número muy limitado de casos, ya que no contamos con los mismos en nuestro medio de trabajo (Servicio de Urgencias de Atención Primaria). Estos autores detectan el mayor número de cálculos en diversos tramos del uréter, en total 499 que representan el $44,12 \%$ de su casuística, nosotros detectamos un total de 25 litiasis de localización ureteral, que representan el 78,12\% del total de las 32 litiasis visualizadas en el Rx de abdomen simple, de estas $12(37,5 \%)$ se localizaban en el uréter inferior, que fue la localización más frecuente (Tabla V).

Un caso a destacar fue un paciente con un riñón ectópico de localización pélvica que debutó con cólicos nefríticos, al que se le visualizó en el rx de abdomen simple múltiples imágenes de densidad cálcica en la pelvis, siendo publicado como caso clínico de forma independiente (29), también mediante el Rx de abdomen simple y la UIV se observó una litiasis de aspecto blando localizada en un divertículo del grupo calicial superior del riñón derecho en una paciente con antecedentes de infecciones urinarias y cólicos nefríticos, igualmente publicado como caso clínico (30).

Con respecto al análisis químico de los cálculos (Tabla VI), en un total de 116 cálculos analizados, 71 fueron de oxalato cálcico mono y dihidratado, representando el 61, 20\%, 25 (21,55\%) eran de oxalato cálcico mas fosfato, $15(12,93 \%)$ de fosfato, $3(2,58 \%)$ de ácido úrico y $2(1,72 \%)$ eran de otras composiciones; en el estudio de Alapont y cols. (2001) (5) se analizan 544 cálculos (58,49\%), 256 $(47,06 \%)$ fueron de oxalato cálcico, $80(14,71 \%)$ de oxalato cálcico mas fosfato cálcico y $78(14,34 \%)$ de ácido úrico, los autores destacan la presencia de 4 casos de cálculos por cistina $(0,74 \%)$, perteneciendo 3 a una misma familia.

Otro estudio realizado con un total de 500 cálculos analizados, un $75 \%$ eran cálculos cálcicos (oxalato cálcico y fosfato cálcico), 13\% cálculos úricos (ácido úrico puro y mixto úrico-cálcicos), 10\% eran cálculos infecciosos (fosfato amónico magnésico, fosfato cálcico, urato amónico), $1 \%$ cálculos de cistina y < 1\% cálculos raros espúreos (25). 


\section{BIBLIOGRAFÍA Y LECTURAS} RECOMENDADAS ( ${ }^{*}$ lectura de interés $y^{* *}$ lectura fundamental)

1. Alonso Dorrego JR, López-Tello García JJ, García Cardoso JV. Dolor Urológico. En: Urgencias en Urología. Manual para residentes, Prof. Luis Resel Esteves, Manuel Esteban Fuertes. Jarpyo Editores, Madrid, 1995. Cap.4, p: 68.

2. Buitrago Ramírez F, Calvo Hueros JI, Bravo Simón B. Estudio y seguimiento del cólico nefrítico. JANO 25, 2005; 1(587): 43-6.

**3. Rousand A, Pedrajas A. Estudio epidemiológico de la urolitiasis en España. Asociación Española de Urología. Grupo de Urolitiasis; 1986.

*4. Sánchez-Martín F M, Millán Rodríguez F, Esquena Fernández S, et al. Incidencia y prevalencia de la urolitiasis en España: Revisión de los datos originales disponibles hasta la actualidad. Actas Urol Esp, 2007; 31(5):511-20.

*5. Alapont Pérez FM, Gálvez Calderón J, Varea Herrero, et al. Epidemiología de la litiasis urinaria. Actas Urol Esp, 2001; 25 (5): 341-9.

6. Whaley L, Wong D. Nursing Care of Infants and Children, ed. 3, página 1070. (C)1987 de C.V. Mosby Company. La investigación se reportó en Wong D, Baker C. Pain in children: Comparison of assessment scales. Pediatric Nursing, 1988; 14(1):9-17.

7. William RE. Long-term survery in 538 patient with upper urinary tract stone. Br J Urol, 1963; 35: 416.

8. Lancina JA, Freire C, Busto L, et al. Sarcoidosis y litiasis urinaria. Arch Esp Urol, 1995; 50: 141.

9. Pieras Ayala E. Litiasis de oxalato cálcico monohidrato papilar y de cavidad: estudio comparativo de factores etiológicos. Localización: http:// www.tdx.cesca.es/TDX-0119105-122301/.

10. Buitrago F, Macías S. Nefrolitiasis. Crisis renoureteral. En: semFYC, editores. Guía de actualización clínica en nefrourología. Barcelona: semFYC 2003; p. 81-9.

11. Curhan GC, Willett WC, Rimm EB, et al. Family history and risk of kidney stone. J Am Soc Nephrol, 1997a; 8:1568-73.

12. Medina-Escobedo M, Mussaret Zaidi, Real-de León E, et al. Prevalencia y factores de riesgo en Yucatán, México, para litiasis urinaria. Salud Pública Mex, 2002; 44:541-5.

13. Coe FL, Parks JH, Moore ES. Familiar idiopathic hypercalciuria. N Engl J Med, 1979; 300: 33740.

**14. Menon M, Resnick, MI. Litiasis urinaria: etiología, diagnóstico y manejo médico. En: Campbell: Urología, Walsh Patrick C, et al. $8^{\mathrm{a}}$ ed., Buenos Aires; Médica Panamericana 2004; cap. 96, p. 3541-4.
15. Prince CL, Scardino PL, Wolan TC. The effect of temperature, humidity and dehydration on the formation of renal calculi. J Urol, 1956; 75: 209.

16. Finlayson B. Renal lithiasis in review. Urol Clin North Am 1974; 1:181-212.

17. Ibarz Navarro JM. Tratamiento médico de la litiasis renal recidivante. En: Litiasis urinaria. Editorial Garsi, S. A. Madrid 1999; p115.

18. Brockis JC, Levit A, Cruthers SM. The effects of vegetable and animals protein diets on calcium, urate and oxalate excretion. Br J Urol, 1982; 54: 590-3.

19. Nagen GR, Seebode JJ, Samady AI, et al. Stressful life events and risk of symptomatic kidney stone. Int J Epidemiol 1997; 26: 1017-23.

20. Orloff NA, Stewart AF. Disorders of serum minerals caused by cancer. In Coe FL, Favus MJ (eds): Disorders of bone and Mineral Metabolism. New York, Raven Press, 1992; pp 539-62.

21. Mallette LE, Khouri K, Zengotita H, et al. Lithium treatment incresses intact and midregion parathyroid hormona and parathyroid volume. J Clin Endocrinol Metab, 1989; 68: 654-60.

22. Kobayashi T, Nishizawa K, Mitsumori K, et al. Impact of Date of Onset on the Absence of Hematuria in Patients with Acute Renal Colic The J. Urol, 2003; 170 (4): 1093-6.

23. Ibarz Navarro JM, Lázaro Castillo J, Rioja Sanz LA. Litiasis: sintomatología. En: Jiménez Cruz JF, Rioja Sanz LA, eds. Tratado de Urología. JR Prous Editores; 1993: 741.

24. Cushner HM, Copley JB. Back to basics: The urinalysis: A selected national survey and review. Am J Med Sci 1989; 297: 193.

25. Lancina Martín J.A. Evaluación metabólicomineral del paciente con litiasis urinaria. En: Litiasis urinaria. Grupo Masson, Editorial Garsi, S. A. 1999, Madrid, p.71.

26. Jiménez FJ, Montero. Protocolos de actuación en medicina de urgencias. L. Editorial Mosby/Doyma. Barcelona. 1995.

27. Bloom A, Libson E, Verstandig, et al. The toothroot sign: A characteristic appearance of distal ureteral calculi. Acta Radiol 1988; 39: 212-3.

28. Ramakumar S, Patterson DE, Leroy AJ, et al. Prediction of stone composition from plain radiographs: A prospective study. J Endourol, 1999; 13: 397-401.

29. Hermida Pérez JA, Del Corral Suarez T, Cerdeiras Martinez G, et al. Riñón derecho ectópico hidronefrótico, con litiasis de localización pélvica, presentación de un caso. Arch Esp Urol, 1998; 51 (3): 289-93.

30. Hermida Pérez JA, Guerra Abrante P, Hernández Guerra JS, et al. Litiasis de aspecto blando, alojada en un divertículo calicial del riñón derecho. Presentación de un caso y conducta terapéutica. Arch. Esp. Urol. 2004; 57 (1): 72-5. 